\title{
A criança com constipação intestinal
}

\author{
Izaura Ramos Assumpção ${ }^{1}$ \\ Mestre em Medicina pelo Hospital das Clinicas da Faculdade de Medicina da Universidade de \\ São Paulo \\ Titulo de Especialista em Pediatria com Área de atuação em Gastroenterologia Pediatrica \\ Professora responsável pelo curso de especialização em Gastroenterologia do CAEPP \\ Médica Gastroenterologista e do Pronto Socorro do Hospital Infantil Sabará
}

\section{Resumo}

O artigo aborda o conceito de constipação intestinal e as suas causas. Tem como objetivo alertar o pediatra geral sobre a constipação intestinal na infância.

Palavras chave: constipação intestinal, infância, pediatria

Assumpção, Izaura Ramos. "A criança com constipação intestinal", in Anais do 20. Congresso Internacional Sabará de Especialidades Pediátricas

\footnotetext{
${ }^{1}$ Mesmas informações
} 
A constipação intestinal é queixa comum na faixa etária pediátrica e estudos referem frequências que variam de 17 a 36\% entre os lactentes e escolares e de até 22,3\% entre os adolescentes. Cerca de $60 \%$ das crianças iniciam o quadro no primeiro ano de vida. Outro dado que chama atenção é o tempo médio entre o início dos sintomas e a primeira consulta com gastroenterologistas pediátricos, que é de 1 ano. A amplitude de prevalência da constipação intestinal na população pediátrica geral decorre de variações dos hábitos alimentares, diferenças regionais e principalmente da falta de uniformidade dos critérios adotados para caracteriza-la.

Alguns autores tendem a considerar a constipação intestinal como um problema de saúde pública, pois podem acarretar internações para realizações de enemas de esvaziamento de fecaloma, ou distúrbios hidroeletrolíticos decorrentes de uso inadequado de laxantes, além da baixa estima do paciente e alterações graves da dinâmica familiar, causadas pela perda involuntária de fezes na roupa, queixas frequentes de dor abdominal, infecções de trato urinário, prolapso retal e fissuras anais entre outros sintomas.

A constipação intestinal pode ser classificada em:

Orgânica é aquela que acompanha doenças intestinais ou extraintestinais e corresponde a 5 a $30 \%$ dos casos.

Funcional que é definida dentro das doenças gastrointestinais funcionais, segundo os critérios de Roma, correspondendo à grande maioria dos casos.

Oculta que foi introduzida em 1994, por Maffei, para aquelas crianças constipadas que só tiveram seu diagnóstico realizado após procurar o médico por complicações da constipação como, por exemplo escape fecal e ITUs.

A tabela abaixo lista as principais causas de constipação intestinal de causa orgânica

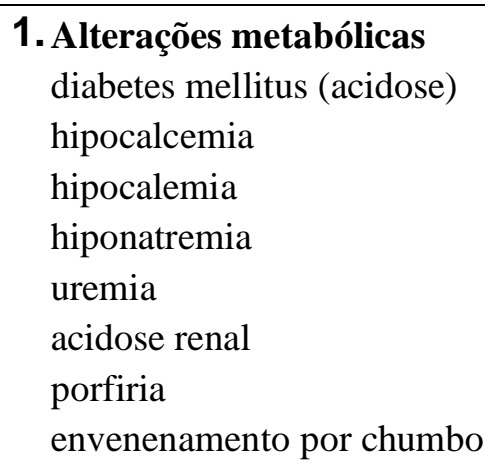

\section{Alterações endócrinas}

pan-hipopituarismo

feocromocitoma 
hipotireoidismo

hiperparatireoidismo

3. Alterações do sistema neuromuscular

doença de Hirschsprung

pseudo-obstrução intestinal

paraplegia

paralisia cerebral

espinha bífida

meningomielocele

doenças do colágeno

tumor de medula

trauma de medula

síndrome de Down

4. Alterações nutricionais

raquitismo

anemia

desnutrição

subalimentação

5. Alterações imunoalérgicas

alergia a proteína do leite de vaca

doença celíaca

6. Alterações estruturais

estenose hipertrófica do piloro

hérnias

volvo

intestino irritável

fissuras,, atresias e estenoses anais

ânus anteriorizado

ânus ectópico

ânus imperfurado

bridas peritoneais

\section{Indução por drogas}

antiácidos (compostos de cálcio e alumínio)

anticolinérgicos (descongestionantes, antiespasmódicos)

anticonvulsivantes

antidepressivos (fenotiazinas)

bismuto

diuréticos

metais (arsênico, chumbo, mercúrio)

sulfato de bário

hematínicos (especificamente Fe)

opiáceos 
São sinais de alerta para investigação de constipação intestinal de causa orgânica:

- Início precoce em menores de 1 mes de idade

- Eliminação mecônio maior de 48h

- História familiar de megacolon congenito

- Sangue nas fezes sem fissura anal

- Falência crescimento

- Vômitos biliosos

- Fístula perianal

- Ânus ectópico

- Agenesia anal ou de reflexo clemastérico

- Tufo de cabelo na coluna sacral

- Desvio de prega glútea

A constipação intestinal crônica funcional definida pelos critérios de ROMA III está baseada nos critérios que se seguem.

Para neonatos e lactentes jovens ( $<4$ anos de idade), pelo menos 2 dos critérios abaixo, por tempo superior a 1 mês

$\square$ menos de 2 evacuações por semana

$\square$ evacuação dolorosa ou com esforço

$\square$ ao menos 1 episódio de incontinência escape fecal

$\square$ fezes grandes em reto ou palpáveis em abdômen

$\square$ fezes volumosas que entopem o vaso sanitário

história de retenção de fezes

Podem ser sintomas concomitantes irritabilidade, diminuição do apetite e saciedade precoce, mas que desaparecem com evacuação em grande quantidade.

Para crianças maiores de 4 anos e adolescentes, pelo menos 2 dos critérios abaixo há pelo menos 8 semanas

$\square$ menos de 2 evacuações por semana

$\square$ evacuação dolorosa ou com esforço

$\square$ ao menos 1 episódio de incontinência escape fecal

$\square$ fezes grandes em reto ou palpáveis em abdômen

$\square$ fezes volumosas que entopem o vaso sanitário

$\square$ postura de retenção ou retenção voluntária de fezes

As manifestações clínicas da constipação intestinal variam com o tempo de constipação e com a idade da criança. As mães muitas vezes não reconhecem a constipação intestinal de seu filho, e as crianças por sua vez, exceto pelo fato de se queixarem de dor abdominal, não a relacionam. O simples questionamento sobre o número de evacuações por dia, não deve satisfazer o pediatra. A característica das fezes, quanto ao calibre, a dificuldade de eliminação, a dor anal ou a eliminação com esforço devem ser questionadas e valorizadas. Dessa forma, o uso da Tabela de Bristol, que caracteriza o aspecto das evacuações também pode auxiliar no reconhecimento da constipação. 
Constipation in children

Abstract: Intestinal constipation is a commun problem in pediatric clinic. This article presents a definition of intestinal constipation in children and the basal causes Keywords: constipation, infants, children

References

Clinical Practice Guideline: Evaluation and Treatment of Constipation in Children: Summary of Updated Recommendations of the North American Society for Pediatric Gastroenterology, Hepatology and Nutrition. JPGN 43:405Y407.2006.

Clinical Practice Guideline: Evaluation and Treatment of Constipation in Infants and Children: Recommendations of the North American Society for Pediatric Gastroenterology, Hepatology and Nutrition. JPGN 43:e1Ye13. 2006.

Cucchiara S; Gobio Casali I; Iacono G; SanfilippoM; Torre G. Bowel frequency in healthy children. Acta Paediatr Scand 78: 682-684. 1989

Del Ciampo IRL. Constipação intestinal crônica na infância: prevalência, diagnóstico e valorização de seus sinais e sintomas e unidade básica de saúde. Dissertação de Mestrado, Faculdade de Medicina de Ribeirão Preto da USP, Ribeirão Preto. p.1-116, 1999.

Evaluation and Treatment of Functional Constipation in Infants and Children: Evidence-Based Recommendations. From ESPGHAN and NASPGHAN. M.M. Tabbers, C. DiLorenzo, M.Y. Berger, C. Faure, M.W. Langendam, S. Nurko, A. Staiano, Y. Vandenplas, and M.A. Benninga. JPGN 58: 258-274. 2014

Koda Y . Obstipação intestinal crônica. In Barbieri D; Koda YKL. Doenças Gastroenterologicas em Pediatria. Ateneu. 1996.

Maffeil HVL; Moreira FL; Oliveira Jr WM; Sanini V.Prevalência de constipação em escolares do ciclo básico. Pediatr (Rio de Janeiro) 73: 340-344, 1997.

Morais MB \& Maffei HVL. Constipação intestinal. J Pediatr (Rio de Janeiro.) 76: 147-156, 2000. Supl.2 
Oliveira JN; Tahan S; Goshima S; Fagundes-Neto U; Morais MB Prevalência de constipação em adolescentes matriculados em escolas de São José dos Campos, SP, e em seus pais Arq. Gastroenterol.43: (1) São Paulo . 2006

Weaver LT. Bowel habit from birth to old age. J Pediatr Gastroenterol Nutr 7: 637$640,1988$.

Moreira FL; Coelho CAR; Maffei HVL. Constipação intestinal crônica em crianças atendidas no ambulatório de gastroenterologia infantil da Faculdade de Medicina de Botucatu-UNESP (1977- 1980). J Pediatr (Rio de Janeiro) 57: 62-65, 1984.

Motta Mefa; Silva GAP. Constipação intestinal crônica funcional na infância: diagnóstico e prevalência em uma comunidade de baixa renda. J Pediatr (Rio de Janeiro) 74: 451-454, 1998 